\title{
Methods of synthesizing qualitative research studies for health technology assessment
}

\author{
Nicola Ring, Ruth Jepson \\ University of Stirling \\ Karen Ritchie \\ Healthcare Improvement Scotland
}

Objectives: Synthesizing qualitative research is an important means of ensuring the needs, preferences, and experiences of patients are taken into account by service providers and policy makers, but the range of methods available can appear confusing. This study presents the methods for synthesizing qualitative research most used in health research to-date and, specifically those with a potential role in health technology assessment.

Methods: To identify reviews conducted using the eight main methods for synthesizing qualitative studies, nine electronic databases were searched using key terms including meta-ethnography and synthesis. A summary table groups the identified reviews by their use of the eight methods, highlighting the methods used most generally and specifically in relation to health technology assessment topics.

Results: Although there is debate about how best to identify and quality appraise qualitative research for synthesis, 107 reviews were identified using one of the eight main methods. Four methods (meta-ethnography, meta-study, meta-summary, and thematic synthesis) have been most widely used and have a role within health technology assessment. Meta-ethnography is the leading method for synthesizing qualitative health research. Thematic synthesis is also useful for integrating qualitative and quantitative findings. Four other methods (critical interpretive synthesis, grounded theory synthesis, meta-interpretation, and cross-case analysis) have been under-used in health research and their potential in health technology assessments is currently under-developed. Conclusions: Synthesizing individual qualitative studies has becoming increasingly common in recent years. Although this is still an emerging research discipline such an approach is one means of promoting the patient-centeredness of health technology assessments.

Keywords: Qualitative research, Patient-centered care, Review literature, Health technology assessment, Synthesis

\footnotetext{
All authors attest that (1) Each named author contributed to both the conception/design and/or analysis/interpretation of the project and the writing of the paper; (2) Each has approved the version being submitted; and (3) The content has not been published nor is being considered for publication elsewhere (although a longer report by the authors on the same topic has been published by NHS Quality Improvement Scotland). We acknowledge input from staff at NHS Quality Improvement Scotland (NHS QIS) (now Healthcare Improvement Scotland) and the University of Aberdeen who contributed to the full report on which this study is based. This work was funded by Healthcare Improvement Scotland including a consultancy fee to N.R. and R.J. to author the full report (18). K.R. is employed by Healthcare Improvement Scotland.
} 
Table 1. Use of Qualitative Synthesis in HTAs (10)

In HTAs, qualitative synthesis can:

- Contribute to decision makers having the best possible evidence base

- Help to assess core patient aspects in relation to a given HTA

- Be used to assess whether there is a need for primary research

- Be used to gain new insight into relevant patient and/or organisational aspects

- Be used to make a generalization.

To ensure that the needs, preferences, and experiences of service users are taken into account when developing and evaluating health technologies or care delivery models (4), health technology assessments (HTA) should take a patientcentered approach. One way of achieving this is by synthesizing individual qualitative research studies.

Qualitative research arose from several disciplines and traditions with various underpinning philosophies (e.g., phenomenology, see Supplementary Glossary, which can be viewed online at www.journals.cambridge.org/thc2011029) and data can be analyzed in different ways (e.g., using grounded theory). There is, therefore, no single approach or definition (14). While the underpinning philosophy may differ, qualitative research has some common characteristics. Generally, it refers to research exploring peoples' experiences and understandings - the "hows" and "whys" of a social phenomena (13). As such, qualitative research has a role in understanding the views of patients, or carers, of what it is like living with a particular condition and their experiences of health services or technologies.

Common qualitative data collection methods are observation, interviews, and focus groups, which produce textual data rich in meaning and insight. Data analysis usually involves inductive reasoning processes (hypothesis generating) to interpret and structure data/findings (23). Influences on the qualitative data analysis process include the researcher(s) theoretical or philosophical "lens" through which they collect and understand their data (23). Such influences, therefore, need to be considered when synthesizing qualitative research and assessing validity. Although the results from one qualitative study may be difficult to generalize, a syntheses of all the relevant qualitative studies can identify common themes and any divergent views.

The synthesis of qualitative studies has been encouraged recently, for example, within HTA (10) and systematic reviews of effectiveness (17). Tables 1 and 2 suggest ways in which such syntheses can contribute to these processes. There is, however, no single approach to the identification and synthesis of health(-related) qualitative research and several methods are available. In our opinion, this range can appear over-whelming, even daunting, for those new to this field.

This study aims to provide some clarity by presenting the main methods for synthesizing the qualitative literature with a potential role in the HTA process. Although meth-
Table 2. Use of Qualitative Research in Intervention Reviews (17)

Qualitative research can contribute to intervention reviews by:

- Informing reviews by using evidence from qualitative research to help define and refine the question, and to ensure the review includes appropriate studies and addresses important outcomes

- Enhancing reviews by synthesizing evidence from qualitative research identified while looking for evidence of effectiveness

- Extending reviews by undertaking a search to specifically seek out evidence from qualitative studies to address questions directly related to the effectiveness review

- Supplementing reviews by synthesizing qualitative evidence within a standalone, but complementary, qualitative review to address questions on aspects other than effectiveness.

ods are discussed separately, in practice they overlap and are inter-related, with researchers often adapting principles of a specific method(s). Areas of debate also still exist, for example, whether it is appropriate, or feasible, to synthesize qualitative studies conducted from different epistemological perspectives (11). We present our interpretation of this complex body of literature.

\section{METHODS}

Having previously reviewed an extensive, but not exhaustive, body of literature to report on the eight main methods for synthesizing qualitative research (18), we now needed to identify reviews using each of these approaches (18). Nine electronic databases were searched during 2010 using relevant search terms (see Table 3 for details and inclusion criteria) to identify reviews conducted using: meta-ethnography, thematic synthesis, meta-study, meta-summary, critical interpretive synthesis, qualitative cross-case analysis, grounded theory synthesis, and meta-interpretation. For each of these methods, the titles and abstracts of potential reviews were screened by R.J. and N.R. working independently and then collaboratively to compare their findings. In cases of uncertainty, the full paper was retrieved. Each included review was then also broadly categorized (Table 3 ) according to its primary focus that is, whether it reported experiences and/or views on: (i) a disease, clinical condition or health(-related) situation; (ii) health services or technologies; (iii) other health and/or social topics.

After identifying the number and type of reviews conducted using each method, we created a summary table to provide a visual representation of which of these methods of qualitative synthesis were being used and with what focus (Supplementary Table 1, which can be viewed online at www.journals.cambridge.org/thc2011029).

\section{RESULTS}

Identifying reviews conducted using these eight methods was challenging. There were many cases where the used 
Table 3. Identifying Reviews Which Synthesised Qualitative Health Research Using One of the Eight Methods

\section{Databases searched (from 1980 until Dec 2010):}

Medline, DARE, HTA, CCTR, CDSR, British Nursing Index, PsycINFO, CINAHL and EmBase

\section{Search terms:}

(meta ethnograph* or meta-ethnograph* or metaethnograph* or Noblit) or (Meta summary or metasummary or meta-summary) or (Meta study or meta-study or metastudy) or (critical interpretive synthesis) or (thematic adj3 synthesis) or (meta interpretation or meta-interpretation or met interpretation) or (grounded theory synthesis or (grounded theory adj5 synthesis)) or ((cross study) and (synthesis or review) and (qualitative))

Search results:

199 references of possible inclusions retrieved (all English language).

\section{Inclusion criteria:}

Reviews were included if they specifically referred to using meta-ethnography, thematic synthesis, meta-study, meta-summary, critical interpretive synthesis, qualitative cross-case analysis, grounded theory synthesis or meta-interpretation. Screening was based on title and abstract. If it was unclear which method was used, the full paper was retrieved for details about methods and study design. Disagreements about included reviews were resolved through team discussion. Unpublished and published reviews were included.

\section{Exclusion criteria:}

Primary studies, e.g. those using meta-ethnographic methods, were excluded as were theoretical or methodological papers. Review topics which did not address our three categories e.g. school education. Titles/abstracts not in English.

\section{Screening outcome:}

107 reviews were considered to meet the inclusion criteria.

\section{Categorizing review topics:}

Decisions were made by RJ and NR - based on the title and abstract - regarding whether a review focused on:

a) a disease or clinical condition/health situation, e.g., pregnancy, diabetes

b) a health service or health technology, e.g., medicine use, barriers to clinical services/service delivery

c) another health or social issue, e.g., poverty, domestic violence.

method of qualitative synthesis was not explicitly articulated within the review title and/or abstract. Terminology was often used inconsistently and interchangeably. The term "meta-synthesis" was frequently used as a synonym for qualitative synthesis regardless of which method was used. "Meta-synthesis" could also refer to the whole review process including study identification, quality assessment and data analysis or specifically refer to the data synthesis element. Nonetheless, across the eight methods for synthesizing qualitative studies, we identified 107 reviews (Table 4, Supplementary Table 1).

Use of the different methods across these 107 reviews varied. Table 4 demonstrates that reviews using critical interpretive synthesis, meta-interpretation, qualitative cross-case analysis and grounded theory synthesis were found infre- quently. As the potential of these methods has yet to be fully established in the context of HTA, these are not discussed further here and readers are referred elsewhere for details (18). Meta-ethnography (16), thematic synthesis (22), and metastudy $(23,24)$ were the most used methods (Table 4$)$, especially on topics relating to health services/technologies and, whereas the number of reviews using meta-summary (20) were small, this method is of relevance to HTA. As such, we consider meta-ethnography, thematic synthesis, meta-study, and meta-summary to have a role in HTA, and these are outlined below with illustrative examples. Readers are referred to Supplementary Table 1 for references of individual reviews and the related theoretical guidance are recommended reading (18).

Meta-ethnography - derived from the work of Noblit and Hare (16) - is currently the most common method for synthesizing qualitative health(-related) research-reflected in our finding of 63 reviews using this method. Metaethnography involves seven steps which, by bringing together findings from individual interpretive accounts, produces a new interpretation (16). To synthesize these findings, metaethnography uses a variety of approaches including "reciprocal translation analysis" and "line-of-argument synthesis" (16).

Most reviews using meta-ethnography were conducted in the 2000s by various healthcare disciplines. Twenty-nine reviews explored perceptions and experiences of disease and other clinical issues including eating disorders, diabetes, pregnancy, and childbirth. Twenty-one reviews explored views on health services/technologies including a range of medications/therapies. A further 13 reviews focused on health/social topics such as poverty and domestic violence (Supplementary Table 1).

One example (12) of interest to HTA reviewers aimed to enhance understanding of patients' experience of antidepressants. Sixteen papers met the inclusion criteria and were quality assessed before synthesis. Two groups of papers were identified-those which focused on the patient-doctor decision-making process and those with a focus on patients' self-concept and notions of stigma (12). The reviewers found that the initial diagnosis of depression may be associated with feelings of relief or despair and that patients' preferences for engaging in decision making during their illness is a dynamic process. To improve adherence to advice regarding medication use during depression, it was suggested that GPs should take into account patients' changing situations and sense-of-self during their treatment (12).

Thematic synthesis is often (but not exclusively) used for analyzing qualitative data alongside quantitative data synthesis. Initially developed by researchers from the EPPI-Center, it addresses questions around "what works," primarily in relation to health promotion interventions. Reviewers can synthesize qualitative and quantitative research separately then integrate their findings (21). Thomas and Harden's (22) method develops analytical themes through descriptive synthesis and 
Table 4. Summary of Methods for Synthesizing Qualitative Research and Related Reviews (For Details Including Full References, See Supplementary Table 1)

\begin{tabular}{|c|c|c|c|c|}
\hline \multirow[b]{2}{*}{ Method } & \multirow[b]{2}{*}{$\begin{array}{l}\text { Number of published } \\
\text { reviews identified }\end{array}$} & \multicolumn{3}{|c|}{ Focus of reviews } \\
\hline & & $\begin{array}{l}\text { Disease or clinical } \\
\text { condition/health } \\
\text { situation }\end{array}$ & $\begin{array}{l}\text { Health services or } \\
\text { technologies }\end{array}$ & $\begin{array}{l}\text { Other health and/or } \\
\text { social topics }\end{array}$ \\
\hline Meta-ethnography & 63 reviews & $\begin{array}{l}29 \text { reviews including } \\
\text { topics such as } \\
\text { women's health, } \\
\text { diabetes, dementia, } \\
\text { long-term conditions. }\end{array}$ & $\begin{array}{l}21 \text { reviews including views } \\
\text { on health interventions for } \\
\text { women, medications, } \\
\text { treatment, health } \\
\text { professional practice e.g. } \\
\text { triage }\end{array}$ & $\begin{array}{l}13 \text { reviews including } \\
\text { topics such as } \\
\text { workplace health } \\
\text { issues, domestic } \\
\text { violence and the } \\
\text { family }\end{array}$ \\
\hline Thematic synthesis & 15 reviews (17 papers) ${ }^{* *}$ & $\begin{array}{l}5 \text { reviews on topics such } \\
\text { as teenage pregnancy } \\
\text { and children's views } \\
\text { of obesity }\end{array}$ & $\begin{array}{l}10 \text { reviews including views } \\
\text { on health professional } \\
\text { practice, health services and } \\
\text { health care, and primary } \\
\text { care/health promotion }\end{array}$ & \\
\hline Meta-study & 13 reviews (14 papers) $)^{* *}$ & $\begin{array}{l}5 \text { reviews on topics } \\
\text { including chronic } \\
\text { illness, fatigue, and } \\
\text { ethnicity and health }\end{array}$ & $\begin{array}{l}7 \text { reviews including views on } \\
\text { spirituality at end-of-life } \\
\text { and decision making }\end{array}$ & $\begin{array}{l}1 \text { review on becoming a } \\
\text { nurse }\end{array}$ \\
\hline Meta-summary & 5 reviews (6 papers) $)^{* *}$ & $\begin{array}{l}1 \text { review (two papers) } \\
\text { on motherhood and } \\
\text { HIV positive mothers }\end{array}$ & $\begin{array}{l}4 \text { reviews including patients' } \\
\text { compliance with } \\
\text { hypertension management } \\
\text { and spiritual care in } \\
\text { end-of-life. }\end{array}$ & \\
\hline Grounded theory synthesis & 3 reviews & 1 review on caring & $\begin{array}{l}1 \text { review of family members } \\
\text { experiences of } \\
\text { life-sustaining treatments } \\
\text { decisions for dying relatives }\end{array}$ & $\begin{array}{l}1 \text { review of experiences } \\
\text { of domestic violence }\end{array}$ \\
\hline Meta-interpretation & 3 reviews & $\begin{array}{l}\text { I review on lay beliefs } \\
\text { about hypertension } \\
\text { and } 1 \text { review of } \\
\text { courage }\end{array}$ & & $\begin{array}{l}1 \text { review on parental } \\
\text { involvement }\end{array}$ \\
\hline $\begin{array}{l}\text { Critical interpretive } \\
\text { synthesis }\end{array}$ & 3 reviews (5 papers) & & $\begin{array}{l}3 \text { reviews including morphine } \\
\text { to treat cancer related pain; } \\
\text { access to primary health } \\
\text { care and access to } \\
\text { healthcare by vulnerable } \\
\text { groups }\end{array}$ & \\
\hline $\begin{array}{l}\text { Qualitative cross-case } \\
\text { analysis }\end{array}$ & 2 reviews & & $\begin{array}{l}1 \text { review of home visiting and } \\
1 \text { review of prenatal } \\
\text { cytogenetic diagnosis }\end{array}$ & \\
\hline
\end{tabular}

${ }^{*}$ Reviews were identified which reported using meta-ethnography, meta-study, meta-summary, thematic synthesis, grounded theory synthesis, meta-
interpretation, critical interpretative synthesis, or cross-case analysis in title or abstract. ** Some reviews are reported in more than one paper by the same authors.

finding of explanations relevant to a particular review question (22). This method was developed to address specific review questions about need, appropriateness, and acceptability of interventions, as well as effectiveness. Peoples' views and experiences are taken into account, and hypotheses that could be tested against the findings of qualitative studies are generated. We identified 15 reviews - 10 of these investigated topics relating to health services/technologies including health promotion, decision making in chronic kidney disease, and the patient-doctor relationship. Five reviews focused on understanding disease/clinical conditions such as lay understanding of cancer risks and mothers experiences of bottle-feeding (Supplementary Table 1).

An example of thematic synthesis of relevance to HTA is a study on adolescent experiences following organ transplantation (25). This study aimed to improve understanding of the psychosocial impact of transplantation from a wide body of research from different time periods, locations, and contexts. Eighteen papers met the inclusion criteria and data were extracted and synthesized from individual results sections to identify descriptive themes. Five themes were identified: redefining identity; family functioning; social adjustment; managing medical demands; and, attitude to the donor (25). 
The reviewers concluded that to ensure adolescent patients are able to deal with the multifaceted nature of their psychological response to transplantation, healthcare teams need to provide them with relevant skills and support (25).

Meta-study involves critical interpretation of existing qualitative research (6). Before synthesis can take place and a new interpretation obtained, three analytical phases are completed-"meta-theory, meta-method, and meta-data analysis" (24). These phases, equating to the analysis of theory, analysis of methods, and the analysis of findings (5), can be conducted concurrently to "provide a unique angle of vision from which to deconstruct and interpret" a body of qualitative literature (6). Once these analytical processes have been completed, meta-synthesis "brings back together ideas that have been taken apart," creating a new interpretation of the phenomenon under investigation (6). Our search identified thirteen reviews mostly published in nursing journals. Seven of these studies were potentially relevant to HTA reviewers for example, those focusing on shared decision making and spirituality in end-of-life care (Supplementary Table 1).

Edwards et al. (8) used meta-study to understand why shared decision making in healthcare was not being implemented and to develop a model of external influences on health information exchange. These reviewers identified three themes: internet information use; cultural differences in intercultural consultations; and the influence of the women's health movement (8). A range of external influences relating to the practitioner and/or patient were included in the model. These influences included receptiveness to patient empowerment, expression of cultural identity and role expectations while health information exchange was assisted where patients already had skills in sourcing and understanding basic health information (8).

Meta-summary uses "quantitatively oriented aggregation of qualitative findings that are themselves topical or thematic summaries or surveys of data" (20). Such summaries can be conducted on their own, or in association with more traditional qualitative synthesis, and can include qualitative and quantitative descriptive findings. Although the least used method presented here-we only identified five reviews on topics such as compliance with hypertension therapy, motherhood and HIV infection (Supplementary Table 1) - it is of relevance to HTA as meta-summary reflects a quantitative perspective and can be used with survey data which is often excluded from some qualitative synthesize due to lack of conceptual depth and richness.

Meta-summary has been used to explore the experiences of parents receiving a positive prenatal test result indicating pregnancy could result in a child with a disability (19). Seventeen qualitative studies of American women and/or their partners who received a positive prenatal test result were synthesized. Once data were extracted, findings were reduced to 39 meta-findings, which were ordered by their relative presence or prominence. The most prominent finding was that positive diagnosis is depicted as the experience of termination with an emphasis on parental choice and decision making (19). The reviewers suggest that healthcare services should support couples receiving a positive prenatal diagnosis to develop a "narrative of choosing" that can allow them to come to terms with their decisions post-testing (19).

\section{DISCUSSION}

One way of incorporating qualitative research and the perspectives of service users within HTAs is through synthesizing individual studies. Although some researchers in certain disciplines, especially nursing, have been synthesizing qualitative studies for some time, this approach has become increasingly common among a wider range of disciplines. The strength of qualitative research is its ability to provide personal insight into the phenomena being investigated but its perceived limitation has been the small number of participants and the inability to generalize findings. Being able to synthesize individual studies to produce a "stronger" body of knowledge has, therefore, become of critical importance if the full potential of qualitative research to inform practice and policy is to be realized.

Qualitative research and the synthesis of individual studies has a considerable role to play in HTA and policy making, as such insight and knowledge is one mechanism for ensuring these processes become more patient/carer-centered. The synthesis of qualitative studies enables reviewers and policy makers to understand what it is like for people to live with a particular long-term condition, experience the effects of medications and/or the process of health service delivery and new technologies. This insight is, therefore, invaluable to those conducting HTA as such knowledge can help explain why particular interventions work, or are unlikely to work, from the perspective of patients/carers-insight that is not possible through quantitative research and systematic review alone. However, there are several issues of relevance to those synthesizing qualitative research.

Searching for qualitative studies is more complex and difficult than searching for quantitative studies (1) and is an under-developed area of qualitative review methodology (9). Some qualitative methods (such as those which mirror the quantitative systematic review approach) aim to be comprehensive, identifying all potential studies but other methodssuch as meta-ethnography - may be more purposive, and aim to reach theoretic saturation rather than identify all relevant studies (17). Use of inclusion criteria also varies depending on the reviewers' underlying philosophical approach. For example, a qualitative synthesis conducted alongside a systematic review of quantitative studies, is likely to have well defined and explicit inclusion criteria (11). Whereas, a standalone synthesis of qualitative studies may include studies based on "conceptual robustness" and theoretical saturation (2), perhaps adopting a more iterative approach to literature searching and screening. 
Quality appraisal of included qualitative studies is not as straightforward as for quantitative studies. There is currently little consensus as to what are the essential criteria for a high-quality qualitative study and over 100 quality appraisal tools are available (17). What constitutes quality in qualitative research is a contested area and part of a bigger debate over the nature of knowledge generation (15). Different disciplines may place a higher value on some aspects of study design such as the theoretical perspective or analytical strategy and there are concerns about excluding less well-conducted studies on the grounds of quality as they may still provide important new insights into a phenomenon (3). Whereas some methods such as thematic synthesis have specific approaches to quality assessment, other approaches such as meta-ethnography are "less committed to the concept" of quality appraisal (5).

Although we found 107 reviews across the eight methods for synthesizing qualitative research, use of these methods varied considerably, especially in topics relating to reviews of health services/technologies. Grounded theory synthesis, meta-interpretation, critical interpretive synthesis, and cross-case analysis have been used less oftengenerally and specifically on topics relating to health services/technologies_-and their usefulness in the context of HTA has yet to be fully established (18). However, metaethnography, meta-study, meta-summary, and thematic synthesis have been more widely used and could have a greater role in HTA.

An earlier review of studies synthesizing qualitative research published between 1988 and 2004 reported that metaethnography was the most frequently used method (7). Our review indicates that meta-ethnography continues to be used most across a range of multi-disciplinary topics including investigations of health services and technologies and, as such, has consolidated its position as the leading method for synthesizing qualitative research. Whereas other methods, for example meta-study, have the potential for broader application they have been used less frequently across a narrower range of topics, often in nursing practice. Those synthesizing qualitative research tend to adapt methods to suit their studies $(7 ; 18)$ and the relevant terminology is used inconsistently and inter-changeably. This has implications for those synthesizing qualitative studies for use in HTA, especially during identification of included studies, as they should not assume original authors/researchers using the same or similar term, share the same definition or understanding of that term in practice. Consequently, a limitation of our study is that we may not have identified all relevant reviews conducted using each method due to ambiguous terminology and/or lack of information about methods in titles and abstracts. There is, therefore, an urgent need for a consensus among qualitative synthesizers regarding such terminology.

Synthesizing qualitative research can ensure the needs, preferences and experiences of patients/carers are reflected within the HTA process in two ways. First, standalone syn- theses of qualitative studies can provide vital contextual information. For example if an HTA is being conducted on a new model of diabetes health service delivery, a qualitative synthesis on the topic of caring for a child with diabetes could help HTA reviewers better understand how that new service may or may not fit with how parents/carers currently manage the condition. Second, findings from qualitative syntheses can be integrated with findings from trial based systematic reviews or other quantitative evidence. This approach can contribute to the HTA process by providing a better understanding of the effectiveness of interventions, such as the seminal example of thematic synthesis which juxtaposed barriers and facilitators to healthy eating by children identified from qualitative studies with findings from evaluated interventions (21).

\section{CONCLUSIONS}

Synthesizing qualitative research is one mechanism for ensuring that patient/carer views and perspectives are incorporated into health service policy making and delivery. There is a considerable body of knowledge on this topic but this literature is complex as several methods exist, the field is still evolving and those synthesizing qualitative research may approach their studies from differing epistemological stances. From the many methods available, meta-ethnography and thematic synthesis appear to be the most useful to HTA reviewers but other methods, including meta-summary, may also be of benefit. Overall, the potential of these methods is yet to be fully realized in terms of using existing qualitative syntheses as contextual information for HTA and through integrating qualitative and quantitative review findings. Nonetheless, those conducting HTA and policy makers must recognize that incorporating the synthesis of qualitative literature into their work can add new insight and value. In particular, understanding that without taking into account the views of patients/carers when health services or technologies are being planned or policy agreed, new developments may not fit with patients'/parents' preferences or needs. Whereas qualitative syntheses can be beneficial to those conducting patient-focused HTAs and quantitative systematic reviews by providing new insight, the challenge is how to routinely incorporate existing syntheses into their work and/or undertake their own such studies within, or alongside, the already time intensive HTA process. Those conducting syntheses of the qualitative literature must also take into account the benefits and limitations of qualitative research generally and debate about critical appraisal and literature searching more specifically (13).

\section{SUPPLEMENTARY MATERIAL}

Supplementary Glossary

Supplementary Table 1

www.journals.cambridge.org/thc2011029 
Ring et al.

\section{CONTACT INFORMATION}

Nicola Ring, MSc, BSc, Dip HV, RGN, RSCN (nicola.ring@ stir.ac.uk), Ruth Jepson, PhD, MSc, GN (ruth.jepson@stir. ac.uk), School of Nursing, Midwifery \& Health, University of Stirling, Stirling FK9 4LA, Scotland, UK.

Karen Ritchie, PhD, MPH, BSc (karenritchie@nhs.net), Healthcare Improvement Scotland, Delta House, 50 West Nile Street, Glasgow G1 2NP, Scotland

\section{CONFLICT OF INTEREST}

Karen Ritchie is employed by Healthcare Improvement Scotland (previously NHS Quality Improvement Scotland). Nicola Ring and Ruth Jepson received consultancy fees for this work from NHS Quality Improvement Scotland.

\section{REFERENCES}

1. Barroso J, Gollop CJ, Sandelowski M, et al. The challenges of searching for and retrieving qualitative studies. West $J$ Nurs Res. 2003;25:153-178.

2. Dixon-Woods M, Agarwal S, Jones D, Young B, Sutton A. Synthesizing qualitative and quantitative evidence: A review of possible methods. J Health Serv Res Policy. 2005;10:45-53.

3. Dixon-Woods M, Sutton AJ, Shaw R, et al. Appraising qualitative research for inclusion in systematic reviews: A quantitative and qualitative comparison of three methods. J Health Serv Res Policy. 2007;12:42-47.

4. Facey K. Patients' perspectives in health technology assessment: A route to robust evidence and fair deliberation. Int $J$ Technol Assess Health Care. 2010;26:334-340.

5. Barnett-Page E, Thomas J. Methods for the synthesis of qualitative research: A critical review. London: Evidence for Policy and Practice Information and Co-ordinating (EPPI) Centre, Social Science Research Unit; 2009.

6. Bondas T, Hall EO. Challenges in approaching metasynthesis research. Qual Health Res. 2007;17:113-121.

7. Dixon-Woods M, Booth A, Sutton AJ. Synthesizing qualitative research: A review of published reports. Qual Res. 2007;7:375422.

8. Edwards M, Davies M, Edwards A. What are the external influences on information exchange and shared decision-making in healthcare consultations: A meta-synthesis of the literature. Patient Edu Couns. 2009;75:37-52.

9. Flemming K, Briggs M. Electronic searching to locate qualitative research: Evaluation of three strategies. J Adv Nurs. 2007;57:95-100.
10. Danish Centre for Health Technology Assessment. DACEHTA Health Technology Assessment Handbook. Copenhagen: Danish Centre for Health Technology Assessment, National Board of Health; 2007.

11. Joanna Briggs Institute. Joanna Briggs Institute Reviewer's Manual. Adelaide, Australia: Joanna Briggs Institute; 2008.

12. Malpass A, Shaw A, Sharp D, et al. "Medication Career" or "Moral Career"? The two sides of managing antidepressants: A meta-ethnography of patients' experience of antidepressants. Soc Sci Med. 2009;68:154-168.

13. Malterud K. Qualitative research: Standards, challenges, and guidelines. Lancet. 2001;358:483-488.

14. Mason J. Qualitative researching. 2nd ed. London: Sage; 2007.

15. Mays N, Pope C. Qualitative research in health care. Assessing quality in qualitative research. BMJ. 2000;320:50-52.

16. Noblit GW, Hare RD. Meta-ethnography: Synthesizing qualitative studies. London: Sage; 1988.

17. Noyes J, Popay J, Pearson A, Hannes K, Booth A. Chapter 20: Qualitative research and cochrane reviews. In: Higgins JPT, Green S, eds. Cochrane handbook for systematic reviews of interventions. Version 5.0.1 (updated March 2011). www.mrcbsu.cam.ac.uk/cochrane/handbook (accessed August 2011).

18. Ring N, Ritchie K, Mandava L, Jepson R. A guide to synthesizing qualitative research for researchers undertaking health technology assessments and systematic reviews. 2011. http://www.healthcareimprovementscotland.org/programmes/ clinical_cost_effectiveness/programme_resources/synth_ qualitative_research (accessed August 2011).

19. Sandelowski M, Barroso J. The travesty of choosing after positive prenatal diagnosis. J Obstet Gynecol Neonatal Nurs. 2005;34:307-318.

20. Sandelowski M, Barroso J. Handbook for synthesizing qualitative research. New York: Springer Publishing Company; 2007.

21. Thomas J, Harden A, Oakley A, et al. Integrating qualitative research with trials in systematic reviews. BMJ. 2004;328:10101012.

22. Thomas J, Harden A. Methods for the thematic synthesis of qualitative research in systematic reviews. BMC Med Res Methodol. 2008;8:45.

23. Thorne S. Data analysis in qualitative research. Evid Based Nurs. 2000;3:68-70.

24. Thorne $\mathrm{S}$. The role of qualitative research within an evidencebased context: Can metasynthesis be the answer? Int J Nurs Stud. 2009;46:569-575.

25. Tong A, Morton RL, Howard K, Craig J. Adolescent experiences following organ transplantation: A systematic review of qualitative studies. J Pediat. 2010;155:542-549. 